\title{
Management of lymph node metastases from an unknown primary site to the head and neck (Review)
}

\author{
SHI MIN ZHUANG, XI-FU WU, JING-JIA LI and GE-HUA ZHANG \\ Department of Otorhinolaryngology-Head and Neck Surgery, The Third Affiliated Hospital of Sun Yat-sen University, \\ Guangzhou, Guangdong 510630, P.R. China
}

Received December 5, 2013; Accepted February 12, 2014

DOI: $10.3892 / \mathrm{mco} .2014 .361$

\begin{abstract}
Cancer of unknown primary site (CUP) is an intriguing clinical phenomenon found in $\sim 3-9 \%$ of all head and neck cancers. It has not yet been determined whether CUP forms a distinct biological entity with specific genetic and phenotypic characteristics, or whether it is the clinical presentation of metastasis in patients with an undetected primary tumor and no visible clinical signs. The treatment of patients with cervical lymph node metastases from CUP remains controversial, due to the lack of randomized clinical trials comparing different treatment options. Consequently, treatment is currently based on non-randomized data and institutional policy. In the present review, the range and limitations of diagnostic procedures are summarized and an optimal diagnostic work-up is recommended. The initial preferred diagnostic procedures include fine-needle aspiration biopsy (FNAB) and imaging. Although neck dissection followed by postoperative radiotherapy is the the most generally accepted approach, other curative options may be used in certain patients, such as neck dissection alone, nodal excision followed by postoperative radiotherapy, or radiotherapy alone. There remains controversy regarding target radiation volumes, ranging from ipsilateral neck irradiation to prophylactic irradiation of all the potential mucosal sites and both sides of the neck. When no primary lesion is identified with imaging and endoscopy in patients without history of smoking and alcohol abuse, molecular profiling of an FNAB sample for human papillomavirus and/or Epstein-Barr virus is required.
\end{abstract}

Correspondence to: Dr Ge-Hua Zhang, Department of Otorhinolaryngology-Head and Neck Surgery, The Third Affiliated Hospital of Sun Yat-sen University, 600 Tianhe Road, Guangzhou, Guangdong 510630, P.R. China

E-mail: zhanggeh@mail.sysu.edu.cn

Key words: cervical lymph node metastases, unknown primary tumor, squamous cell carcinoma, diagnostics, treatment

\section{Contents}

1. Introduction

2. Diagnostic work-up

3. Treatment

4. Conclusion

\section{Introduction}

Cancer of unknown primary site (CUP) is a heterogenous group of malignancies presenting with distant metastases without an identified primary tumor at diagnosis (1). As a clinical entity defined by the exclusion of a primary tumor, the standardized diagnostic work-up includes meticulous medical history and clinical examination using modern diagnostic tools. The estimated incidence of CUP accounts for 3-9\% of all head and neck cancers, with squamous cell histology accounting for $53-77 \%$ of the cases (2-4). Over the last few years, there has been a decrease in the incidence of cervical CUP, due to a more accurate diagnosis of the primary tumor by means of a thorough fibre optic endoscopy of the pharynx and larynx, esophagogastroduodenoscopy, bronchoscopy and advanced imaging technology in the majority of patients (5).

Cervical CUP preferentially affects male patients, aged 55-60 years, with a history of alcohol and tobacco abuse; however, a proportion of CUP cases may include younger non-smokers presenting with human papillomavirus (HPV)-related oropharyngeal cancer. The discrepancies observed in certain series may result from the inclusion of patients with tumors of other histological types (such as undifferentiated carcinomas, lymphomas and melanoma) (5-7). The symptom that commonly prompts initial consultation is a mass in the neck, which accounted for $94 \%$ of the 352 patients in the series reported by Grau et al (5), with pain and weight loss reported in only 9 and 7\%, respectively. In a series of 167 patients reported by Issing et al (4), the incidence of cervical swelling, pain and dysphagia was 100, 9 and 3.6\%, respectively. Typically, the enlarged cervical lymph nodes are located at level II, with bilateral involvement reported in $<10 \%$ of the cases $(2,4,5)$. The clinical $\mathrm{N}$ classification in the majority of cases is $\mathrm{N} 2$, with a median nodal size of $3.5-5 \mathrm{~cm}(2,5,8)$ and the time interval between the appearance of cervical mass and diagnosis is $2-5$ months $(2,4)$. 
Table I. Location of neck lymph nodes and possible primary tumor sites.

\begin{tabular}{lll}
\hline Level & Involved neck lymph nodes & \multicolumn{1}{c}{ Possible primary sites } \\
\hline I & Submandibular & Mouth floor, lips, anterior part of the tongue \\
II & Jugulodigastric/upper jugular & Epipharynx, base of tongue, tonsils, nasopharynx, larynx \\
III & Middle jugular & Supraglottic larynx, inferior pyriform sinus, post-cricoid region \\
IV & Inferior jugular & Hypopharynx, subglottic larynx, thyroid, esophagus \\
V & Supraclavicular & Lungs, thyroid, breast, gastrointestinal system \\
\hline
\end{tabular}

The optimal therapeutic management of patients with CUP remains controversial, due to the absence of randomized studies comparing different treatment options. Therefore, treatment is mainly based on non-randomized evidence and institutional policy. Recommendations include surgery alone (9), limited field radiotherapy and extensive prophylactic irradiation of all the potential mucosal sites and both sides of the neck, with or without concomitant chemotherapy (10-12). However, the morbidity of these combined treatments may be high, decreasing the quality of life of relatively young patients. No randomized or prospective studies are currently available to specifically support these approaches and such a study may be difficult to undertake, as this disease is rare.

In this review, only patients with the squamous cell histotype are discussed, as patients with other histological types may undergo different treatment and have a different prognosis. The aim of the present review was to revise the diagnostic work-up of this entity, including molecular biology, and to better define the multidisciplinary approach of patients with cervical node metastases from CUP, including the extent of neck surgery, the volume and dose of radiation and the addition and type of systemic therapy.

\section{Diagnostic work-up}

The diagnostic approaches in patients with CUP are first aimed at establishing the histopathological type of the tumor and, second, at detecting the primary tumor site. These two objectives are interdependent, as histopathological diagnosis guides the search for the primary site and the diagnostic work-up determines the $\mathrm{N}$ and $\mathrm{M}$ classification of the disease. Therefore, the diagnostic manoeuvres include physical examination, fine-needle aspiration biopsy (FNAB), imaging studies, endoscopy and molecular biology studies.

Physical examination. A painless and unilateral cervical mass is the most common clinical presentation. The site of palpable cervical lymphadenopathy may be useful in suggesting the possible primary tumor site. In patients with a squamous cell histotype, the jugulodigastric and midjugular lymph nodes are most commonly involved, whereas metastatic adenocarcinoma is more frequently diagnosed in the low cervical or supraclavicular areas. In addition, based on the metastatic lymph node level, several possible sites of the primary tumors may be predicted (13) (Table I). The most commonly involved level of lymph node involvement is level II (30-50\%), followed by levels I and III (10-20\%) and levels IV and V (5-10\%).
$F N A B$. FNAB is most commonly used as the first-step diagnostic procedure to establish malignancy. This is an efficient, minimally invasive and cost-effective diagnostic method, with a negligible risk of seeding tumor cells along the needle track (14). The diagnosis is usually established with routine histological staining, supplemented by immunohistochemical analysis. Provided this approach is conducted by an experienced histopathologist, it may achieve a diagnostic sensitivity of $83-97 \%$ and a specificity of $91-100 \%$ for metastatic lesions $(14,15)$. Repeat FNAB, core or open biopsy may be required in cases of uncertain or non-diagnostic histology. However, the value of performing an open biopsy remains controversial, due to the high incidence of local recurrence. Martin and Morfit (16) described open biopsy as 'ill-advised and needless surgery'. By contrast, no adverse effects regarding neck disease control or survival were reported in studies of open neck lymph node biopsy, provided that the biopsy was followed by definitive treatment in the form of radiotherapy or comprehensive neck dissection (17). In view of these data, it may not be imperative to perform an immediate neck dissection when an open biopsy frozen sections reveal metastatic cancer in the excised node. Of note, an open neck lymph node biopsy should not be performed prior to the completion of a thorough clinical, radiological and endoscopic search for the primary tumor.

Imaging. Computed tomography (CT) or magnetic resonance imaging (MRI) of the neck may be performed in order to determine the extent of the disease. The aim of performing imaging studies in such patients is, first, the detection of the primary site in the head and neck region and, second, the staging evaluation of the lymph node status prior to any locoregional treatment. Imaging should be performed prior to any invasive procedure or treatment, in order to avoid diagnostic misinterpretation. If the lymph node is situated in levels IV or V, a chest/abdominal/pelvic CT is recommended. The extent and location of cervical disease and its association with neighboring structures, the presence of extracapsular extension and the status of the retropharyngeal and contralateral neck nodes should be investigated. In the search for a primary tumor in the head and neck, a CT scan may be either complemented or supplanted by MRI with gadolinium contrast, which exhibits superior soft tissue resolution, particularly for the evaluation of the nasopharynx or oropharynx. The potential of CT, MRI or both to detect a primary tumor ranges from 9.3 to $23 \%(3,18,19)$, rising to $60 \%$ when suspicious radiological findings direct subsequent endoscopic biopsies (20). 
Over the last 2 decades, positron emission tomography (PET) has also been used in patients with CUP. A review of 16 studies using fludeoxyglucose $\left({ }^{18} \mathrm{~F}-\mathrm{FDG}\right)$-PET reported a diagnostic accuracy in detecting the primary tumor site of $24.5 \%$, while sensitivity and specificity were 88 and $75 \%$, respectively $(21,22)$. A disadvantage of FDG-PET is its lack of anatomical information, which may impede precise localization of the FDG accumulation. Therefore, the application of combined FDG-PET/CT or MRI may be of greater value in the detection of the primary site. Several studies using FDG-PET/CT reported identification of the primary tumor in $48-73 \%$ of the cases and modification of treatment plans in almost $30 \%$ of the patients (23). Kwee and Kwee (24) published a meta-analysis of 11 studies, including 433 patients with CUP who underwent PET/CT; the overall primary tumor detection rate was $37 \%$, with $84 \%$ sensitivity and $84 \%$ specificity. In 4 of those studies, only patients with CUP were enrolled and the primary tumor detection rate was $28-57 \%$, whereas sensitivity and specificity were $70-100 \%$ and $73-100 \%$, respectively. In 3 of the trials comparing the utility of PET/CT vs. PET, the authors reported that, although PET/CT identified more primary sites compared to PET, these differences were not statistically significant. Overall, PET/CT was reported to modify therapy in $20-60 \%$ of the cases.

Molecular studies. Better insight into the etiology and pathogenesis of head and neck tumors has led to the development of specific diagnostic tests and effective therapeutic strategies. Molecular studies have the potential to further improve diagnosis. Among head and neck cancers, the Epstein-Barr virus (EBV) genome has been identified only in nasopharyngeal carcinoma (25). As a result, the identification of the EBV genome in a metastatic cervical node may lead to the subsequent identification of an unsuspected primary site within the nasopharynx. In situ hybridization for EBV-encoded RNA or polymerase chain reaction for EBV genomic DNA may be performed on tissue obtained by FNAB and should be considered, particularly for young patients with poorly differentiated squamous cell carcinoma histology in a cervical lymph node (25). High-risk HPV has been advocated as a major etiological factor for a subset of head and neck squamous cell carcinomas. These carcinomas are considered to exhibit a special predilection for the oropharyngeal tonsils and are characterized by non-keratinizing basaloid morphology and a strong reactivity to p16 immunostaining. Identifying HPV DNA or RNA in the lymph nodes may thus provide a means for localizing the primary tumor site in the oropharynx (26). These molecular findings used in conjunction with histology, clinical history and radiological findings, may be used to distinguish the primary site of cervical lymph node metastases of CUP of the head and neck.

Endoscopy with biopsy. A definitive evaluation of the unknown primary site may be performed using endoscopy of the upper aerodigestive tract (UADT) with the patient under general anesthesia, assisted by careful palpation of accessible regions and directed biopsies of clinically or radiologically suspicious areas. Bronchoscopy is recommended when there is an abnormality of the lung on chest imaging (20). The likelihood of discovering a primary tumor by endoscopy correlates with the presence of suspicious findings on preliminary examination. When routine diagnostic work-up fails to identify the primary tumor, subsequent endoscopy may be successful in $29.2 \%$ of the cases, as reported by Cianchetti et al (20). Repeated endoscopy is required only when the suspicious site was not adequately biopsied during the first procedure.

The tonsillar fossa and the base of the tongue are considered to be one of the most common sites of a hidden primary tumor in patients with CUP. Although the true incidence remains unknown, it is estimated to be $18-40 \%$. The tonsils have also been incriminated as the site of occult primary tumors, particularly in patients bearing head and neck tumors associated with chronic HPV infection of the upper aerodigestive mucosa. It was previously suggested that directed random biopsies and unilateral or bilateral tonsillectomy should be included in the screening for detection of the occult primary tumor (27). In the absence of a visible or palpable lesion, Waltonen et al (28) reported that tonsillectomy may result in a significantly higher likelihood of detecting an occult tonsillar tumor compared to deep tonsil biopsy. The evidence supporting bilateral tonsillectomy is less convincing, although the detection rates of a primary tumor site in the contralateral tonsil were reported to be $10-23 \%$ (27-29).

According to data reported in the literature, in an optimal scenario, the diagnostic work-up should be conducted in 4 steps: i) Patient history, clinical examination with UADT assessment by multiple examiners and modern imaging using contrast-enhanced CT or MRI as the method of choice. The added value of FDG-PET/CT to the conventional work-up has yet to be determined. ii) Tissue diagnosis preferably obtained by a FNAB; when the FNAB is non-diagnostic, it should be repeated (image-guided). A core needle biopsy is recommended after multiple inconclusive FNABs followed by excisional biopsy of the enlarged node. Appropriate immunohistochemical examinations allow tissue characterization of the tumor in the vast majority of the patients. iii) Endoscopy under general anesthesia with ipsilateral tonsillectomy and directed biopsy. Optimal imaging information of the potential sites of the hidden primary tumor is crucial, as this guides mucosal sampling. Reversing the order of examinations increases the rate of false-positive findings on CT or PET. A second endoscopy is required only when a suspicious mucosal site is inadequately biopsied during the previous procedure. iv) Molecular studies. To obtain optimal information predicting the primary site, HPV and/or EBV immunostaining of the FNAB sample obtained from the enlarged cervical lymph node is required, particularly in cases where no suggestive findings are recorded on imaging and endoscopy and/or in patients without a history of smoking and alcohol abuse.

\section{Treatment}

The optimal therapeutic management of patients with cervical CUP remains controversial, due to the absence of randomized studies comparing different treatment options. The type of treatment may be individualized depending on patient age, tumor site, histology and extent of metastatic lymph node involvement. Generally, these patients should be treated with aggressive multimodal therapy, similar to patients with locally advanced head and neck cancer. 
The majority of authors recommend surgical removal of the neck disease, followed by comprehensive postoperative radiotherapy (5,30-32). Surgery alone is not recommended, except for patients with pN1 or N2a neck disease in level I, with no extracapsular extension. The locoregional control rates were reported to be $80-90 \%$, with a median nodal recurrence rate of $\sim 34 \%$ and a 5 -year overall survival rate of $\leq 65 \%$ (33). Treating a metastatic lymph node in the neck from CUP requires complying with 2 requirements: i) the surgical procedure must be oncologically sound and the damage inflicted to the anatomical structures of the neck should be kept to a minimum; and ii) open biopsy of a positive lymph node or isolated adenectomy are generally discouraged, unless there is strong cytological evidence of a lymphoma. However, if the cytology is compatible with squamous cell carcinoma, opening the capsule of an infiltrated lymph node or breaking the lymphatic drainage net may increase the risk of tumor dissemination. Thus, the minimum procedure considered for the surgical treatment of a metastatic lymph node in the neck is selective neck dissection of the affected lymphatic levels. The extent of the dissection is determined by the radiological and clinical findings and must involve the entire lymphatic chain where the metastatic lymph nodes are situated, avoiding an oversized surgical procedure. Radical neck dissection should only be considered in cases with radiological signs or intraoperative findings of extracapsular disease involving the jugular vein or the sternocleidomastoid muscle.

Neck dissection in a patient with an unknown primary site is the final step in the diagnostic effort aiming to locate the primary tumor, as well as the first therapeutic procedure. Therefore, neck dissection in a patient with an unknown primary site must be performed only after the exhaustion of all other available diagnostic methods. The cervical specimen will provide enough tumor tissue to perform full immunohistochemical studies that may eventually yield decisive data regarding the site of origin.

Radiation alone has been extensively used, with acceptable results $(5,12)$. A potential advantage of radiation compared to surgery in this setting is that potential primary sites in the head and neck may be included in the radiation field. Neck dissection following irradiation may be required for nodal residual disease (34). A nodal excision may be sufficient when radical, comprehensive irradiation to the neck and mucosal sites is planned (30). The most frequently used therapeutic approach consists of surgical removal of the neck disease, followed by postoperative radiotherapy to the neck, or to the neck and the involved mucosa. The indications for postoperative radiotherapy in patients with CUP include excisional or incisional biopsy of the neck prior to definitive treatment, extracapsular extension of the tumor and multiple positive lymph nodes. However, primary radiotherapy may be administered to patients with initial stage N2b or N3 disease as a sole treatment, or followed by neck dissection after 4-6 weeks. Patients with large nodes fixed to the adjacent structures and patients with a low performance status and comorbidities may also be treated by primary irradiation.

Although the value of irradiation of the potential primary sites has not been confirmed by randomized studies, several authors reported that mucosal irradiation reduces the emergence of primary tumors and regional recurrence, without however affecting overall survival $(33,35,36)$. A higher 5-year overall survival rate was reported for patients treated with extensive radiotherapy including the neck nodes and pharyngeal mucosa, compared to those treated by more limited volumes (57.6 vs. $24 \%$; $\mathrm{P}<0.01)$. According to the level of the neck affected, the dose commonly administered with standard fractionation (dose per fraction of 1.8-2 Gy) is 65-70 Gy to the involved nodal stations and $50 \mathrm{~Gy}$ for the uninvolved neck and mucosal sites. In case of clinically suspicious mucosal sites, a dose of 60-64 Gy is recommended. The predominant manifestations of acute radiation toxicity include dysphagia and mucositis, particularly in patients treated with combined radiochemotherapy compared to those treated with radiotherapy alone. Xerostomia is the most common late complication of radiotherapy. Other late adverse effects include persisting edema of the larynx or skin, soft tissue fibrosis, necrosis and osteoradionecrosis (33,35-37). Huang et al (38) reported severe (grade 3-4) acute mucositis in 34\%, insertion of a nasogastric feeding tube in $54 \%$ and severe late xerostomia or neck fibrosis in $7 \%$ of the patients. Combined with postoperative complications and post-chemotherapy toxicity, antineoplastic treatment may significantly affect the quality of life, particularly in long-term surviving patients, underlining the significance of advanced radiotherapy techniques, such as 3D conformal and intensity-modulated radiation therapy, regardless of any anticipated benefit on tumor control.

Concurrent chemoradiotherapy in patients with locally advanced squamous cell carcinoma of the head and neck significantly improves response rate and overall survival (39-44). In addition, the combination of platinum-based chemotherapy with cetuximab exhibited increased efficacy as first-line treatment of patients with recurrent or metastatic head and neck cancer (43). The abovementioned results were obtained from large, well-conducted randomized studies published over the last few years. However, there are currently no available randomized reports on the efficacy of chemoradiotherapy in patients with CUP. To the best of our knowledge, only 4 retrospective studies, including $\sim 100$ patients treated with various cytotoxic agents, have been conducted to date. Chemotherapy was administered prior to, during or after radiotherapy and the results were compared to historical controls (43-46).

\section{Conclusion}

Over the past several decades, the substantial improvements in diagnostics have allowed more accurate determination of the extent of the disease in the neck and the systemic spread to distant organs and the identification of the hidden primary tumor in more than half of the patients with CUP presenting as a neck mass. In cases with no primary tumor on initial examinations, the site of the index primary may be predicted with a high level of probability.

There remains the question of whether CUP is different from tumors of known primary site. If we accept the concept of a specific natural history and biology of unknown primary tumors, further research on the biology of CUP should focus on determining whether this group of tumors share unique genetic, chromosomal and/or phenotypic anomalies. It is hypothesized that novel molecular techniques, such as DNA and gene expression profiling as well as proteomics, play a significant role in this research $(47,48)$. 
The optimal extension of radiotherapy volumes remains an open issue. A randomized trial proposed by the European Organization for Research on Treatment of Cancer Radiotherapy Group and the Radiation Therapy Oncology Group, designed to investigate whether the disease-free survival achieved by ipsilateral neck irradiation is equivalent to that achieved by a more extensive irradiation field including mucosal and bilateral neck nodes, was prematurely terminated due to insufficient accrual. The answer to this question may lead to major management decisions for the majority of patients with cervical CUP.

The use of immunohistochemical markers may predict response to treatment, similar to other types of head and neck cancer $(48,49)$. However, the focus of investigation is the natural history of CUP. Insights into the molecular biology of CUP are required in order to identify the cell signalling pathways responsible for primary tumor dormancy and early metastatic spread, leading to the optimal diagnostic and therapeutic management of these patients.

\section{Acknowledgements}

This study was supported by the Natural Science and Doctor Program Fund of Guangdong Province (grant no.S2012040006622) and the InternationalCooperative Program Fund of Guangdong Province (grant no. 2012B050600015).

\section{References}

1. Pavlidis N, Briasoulis E, Hainsworth J and Greco FA: Diagnostic and therapeutic management of cancer of unknown primary. Eur J Cancer 39: 1990-2005, 2003.

2. Strojan P and Anicin A: Combined surgery and postoperative radiotherapy for cervical lymph node metastases from an unknown primary tumour. Radiother Oncol 49: 33-40, 1998.

3. Regelink G, Brouwer J, de Bree R, et al: Detection of unknown primary tumours and distant metastases in patients with cervical metastases: value of FDG-PET versus conventional modalities. Eur J Nucl Med Mol Imaging 29: 1024-1030, 2002.

4. Issing WJ, Taleban B and Tauber S: Diagnosis and management of carcinoma of unknown primary in the head and neck. Eur Arch Otorhinolaryngol 260: 436-443, 2003.

5. Grau C, Johansen LV, Jakobsen J, et al: Cervical lymph node metastases from unknown primary tumours. Results from a national survey by the Danish Society for Head and Neck Oncology. Radiother Oncol 55: 121-129, 2000.

6. D'Souza G, Zhang HH, D'Souza WD, Meyer RR and Gillison ML: Moderate predictive value of demographic and behavioral characteristics for a diagnosis of HPV16-positive and HPV16-negative head and neck cancer. Oral Oncol 46: 100-104, 2010.

7. Moncrieff MD, Martin R, O'Brien CJ, et al: Adjuvant postoperative radiotherapy to the cervical lymph nodes in cutaneous melanoma: is there any benefit for high-risk patients? Ann Surg Oncol 15: 3022-3027, 2008.

8. Chen AM, Farwell DG, Lau DH, Li BQ, Luu Q and Donald PJ: Radiation therapy in the management of head-and-neck cancer of unknown primary origin: how does the addition of concurrent chemotherapy affect the therapeutic ratio? Int J Radiat Oncol Biol Phys 81: 346-352, 2011.

9. Coster JR, Foote RL, Olsen KD, et al: Cervical nodal metastasis of squamous cell carcinoma of unknown origin: indications for withholding radiation therapy. Int J Radiat Oncol Biol Phys 23 : 743-749, 1992.

10. Glynne-Jones RG, Anand AK, Young TE and Berry RJ: Metastatic carcinoma in the cervical lymph nodes from an occult primary: a conservative approach to the role of radiotherapy. Int J Radiat Oncol Biol Phys 18: 289-294, 1990.

11. Weir L, Keane T, Cummings B, et al: Radiation treatment of cervical lymph node metastases from an unknown primary: an analysis of outcome by treatment volume and other prognostic factors. Radiother Oncol 35: 206-211, 1995.
12. Ligey A, Gentil J, Crehange G, et al: Impact of target volumes and radiation technique on loco-regional control and survival for patients with unilateral cervical lymph node metastases from an unknown primary. Radiother Oncol 93: 483-487, 2009.

13. Shah JP: Patterns of cervical lymph node metastasis from squamous carcinomas of the upper aerodigestive tract. Am J Surg 160: 405-409, 1990.

14. Layfield LJ: Fine-needle aspiration in the diagnosis of head and neck lesions: a review and discussion of problems in differential diagnosis. Diagn Cytopathol 35: 798-805, 2007.

15. Pfeiffer J, Kayser L and Ridder GJ: Minimal-invasive core needle biopsy of head and neck malignancies: clinical evaluation for radiation oncology. Radiother Oncol 90: 202-207, 2009.

16. Martin H and Morfit HM: Cervical lymph node metastasis as the first symptom of cancer. Surg Gynecol Obstet 78: 133-159, 1944

17. Colletier PJ, Garden AS, Morrison WH, Goepfert H, Geara F and Ang KK: Postoperative radiation for squamous cell carcinoma metastatic to cervical lymph nodes from an unknown primary site: outcomes and patterns of failure. Head Neck 20: 674-681, 1998.

18. Waltonen JD, Ozer E, Hall NC, Schuller DE and Agrawal A: Metastatic carcinoma of the neck of unknown primary origin: evolution and efficacy of the modern workup. Arch Otolaryngol Head Neck Surg 135: 1024-1029, 2009.

19. Freudenberg LS, Fischer M, Antoch G, et al: Dual modality of ${ }^{18} \mathrm{~F}$-fluorodeoxyglucose-positron emission tomography/computed tomography in patients with cervical carcinoma of unknown primary. Med Princ Pract 14: 155-160, 2005.

20. Cianchetti M, Mancuso AA, Amdur RJ, et al: Diagnostic evaluation of squamous cell carcinoma metastatic to cervical lymph nodes from an unknown head and neck primary site. Laryngoscope 119: 2348-2354, 2009.

21. Rusthoven KE, Koshy M and Paulino AC: The role of fluorodeoxyglucose positron emission tomography in cervical lymph node metastases from an unknown primary tumor. Cancer 101: 2641-2649, 2004

22. Fleming AJ Jr, Smith SP Jr, Paul CM, et al: Impact of $\left[{ }^{18} \mathrm{~F}\right]-2-$ fluorodeoxyglucose-positron emission tomography/computed tomography on previously untreated head and neck cancer patients. Laryngoscope 117: 1173-1179, 2007.

23. Wartski M, Le Stanc E, Gontier E, et al: In search of an unknown primary tumour presenting with cervical metastases: performance of hybrid FDG-PET-CT. Nucl Med Commun 28: 365-371, 2007.

24. Kwee TC and Kwee RM: Combined FDG-PET/CT for the detection of unknown primary tumors: systematic review and meta-analysis. Eur Radiol 19: 731-744, 2009.

25. Lee WY, Hsiao JR, Jin YT and Tsai ST: Epstein-Barr virus detection in neck metastases by in-situ hybridization in fine-needle aspiration cytologic studies: an aid for differentiating the primary site. Head Neck 22: 336-340, 2000.

26. El-Mofty SK, Zhang MQ and Davila RM: Histologic identification of human papillomavirus (HPV)-related squamous cell carcinoma in cervical lymph nodes: a reliable predictor of the site of an occult head and neck primary carcinoma. Head Neck Pathol 2: 163-168, 2008.

27. Kothari P, Randhawa PS and Farrell R: Role of tonsillectomy in the search for a squamous cell carcinoma from an unknown primary in the head and neck. Br J Oral Maxillofac Surg 46: 283-287, 2008.

28. Waltonen JD, Ozer E, Schuller DE and Agrawal A: Tonsillectomy vs. deep tonsil biopsies in detecting occult tonsil tumors. Laryngoscope 119: 102-106, 2009.

29. Koch WM, Bhatti N, Williams MF and Eisele DW: Oncologic rationale for bilateral tonsillectomy in head and neck squamous cell carcinoma of unknown primary source. Otolaryngol Head Neck Surg 124: 331-333, 2001.

30. Mendenhall WM, Amdur RJ, Hinerman RW, et al: Management of the neck including unknown primary tumor. In: Principles and Practice of Radiation Oncology. Halperin EC, Perez CA and Brady LW (eds). Vol 1. Lippincott Williams and Wilkins, Philadelphia, PA, pp1035-1054, 2013.

31. Beldi D, Jereczek-Fosa BA, D'Onofrio A, et al: Role of radiotherapy in the treatment of cervical lymph node metastases from an unknown primary site: retrospective analysis of 113 patients. Int J Radiat Oncol Biol Phys 69: 1051-1058, 2007.

32. Madani I, Vakaet L, Bonte K, et al: Intensity-modulated radiotherapy for cervical lymph node metastases from unknown primary cancer. Int J Radiat Oncol Biol Phys 71: 1158-1166, 2008. 
33. Iganej S, Kagan R, Anderson P, et al: Metastatic squamous cell carcinoma of the neck from an unknown primary: management options and patterns of relapse. Head Neck 24: 236-246, 2002

34. Lopez Rodriguez M, Cerezo Padellano L, Martin Martin M and Counago Lorenzo F: Neck dissection after radiochemotherapy in patients with locoregionally advanced head and neck cancer. Clin Transl Oncol 10: 812-816, 2008.

35. Erkal HS, Mendenhall WM, Amdur RJ, et al: Squamous cell carcinomas metastatic to cervical lymph nodes from an unknown head-and-neck mucosal site treated with radiation therapy alone or in combination with neck dissection. Int $\mathbf{J}$ Radiat Oncol Biol Phys 50: 55-63, 2001.

36. Erkal HS, Mendenhall WM, Amdur RJ, et al: Squamous cell carcinomas metastatic to cervical lymph nodes from an unknown head and neck mucosal site treated with radiation therapy with palliative intent. Radiother Oncol 59: 319-321, 2001.

37. Klop WM, Balm AJ, Keus RB, et al: Diagnosis and treatment of 39 patients with cervical lymph node metastases of squamous cell carcinoma of unknown primary origin referred to Netherlands Cancer Institute/Antoni van Leeuwenhoek Hospital, 1979-1998. Ned Tijdschr Genehieskd 144: 1355-1360, 2000 (In Dutch).

38. Huang CC, Tseng FY, Yeh TH, et al: Prognostic factors of unknown primary head and neck squamous cell carcinoma. Otolaryngol Head Neck Surg 139: 429-435, 2008.

39. Adelstein DJ, Li Y, Adams GL, et al: An intergroup phase III comparison of standard radiation therapy and two schedules of concurrent chemoradiotherapy in patients with unresectable squamous cell head and neck cancer. J Clin Oncol 21: 92-98, 2003.

40. Bernier J, Domenge C, Ozsahin M, et al: Postoperative irradiation with or without concomitant chemotherapy for locally advanced head and neck cancer. N Engl J Med 350: 1945-1952, 2004.
41. Cooper JS, Pajak TF, Forastiere AA, et al: Postoperative concurrent radiotherapy and chemotherapy for high-risk squamous-cell carcinoma of the head and neck. N Engl J Med 350: 1937-1944, 2004.

42. Forastiere AA, Goepfert H, Maor M, et al: Concurrent chemotherapy and radiotherapy for organ preservation in advanced laryngeal cancer. N Engl J Med 349: 2091-2098, 2003.

43. Vermorken JB, Mesia R, Rivera F, et al: Platinum-based chemotherapy plus cetuximab in head and neck cancer. N Engl J Med 359: 1116-1127, 2008.

44. de Braud F, Heilbrun LK, Ahmed K, et al: Metastatic squamous cell carcinoma of an unknown primary localized to the neck. Advantages of an aggressive treatment. Cancer 64: 510-515, 1989.

45. Argiris A, Smith SM, Stenson K, et al: Concurrent chemoradiotherapy for N2 or N3 squamous cell carcinoma of the head and neck from an occult primary. Ann Oncol 14: 1306-1311, 2003.

46. Shehadeh NJ, Ensley JF, Kucuk O, et al: Benefit of postoperative chemoradiotherapy for patients with unknown primary squamous cell carcinoma of the head and neck. Head Neck 28 : 1090-1098, 2006.

47. Pentheroudakis G, Briasoulis E and Pavlidis N: Cancer of unknown primary site: missing primary or missing biology? Oncologist 12: 418-425, 2007.

48. Pavlidis N, Pentheroudakis G and Plataniotis G: Cervical lymph node metastases of squamous cell carcinoma from an unknown primary site: a favourable prognosis subset of patients with CUP. Clin Transl Oncol 11: 340-348, 2009.

49. Seiwert TY and Cohen EE: Targeting angiogenesis in head and neck cancer. Semin Oncol 35: 274-285, 2008. 